\title{
Efecto de cinco niveles de balance electrolítico dietario en el crecimiento, características de carcasa y metabolitos de suero sanguíneo del cuy (Cavia porcellus)
}

\author{
Effects of five levels of dietary electrolyte balance on growth, carcass \\ characteristics and blood serum metabolites of guinea pig (Cavia porcellus) \\ Manuel Paredes ${ }^{1,4}$, José Mantilla ${ }^{1}$, Irma Bustamante ${ }^{1}$, Joe Mantilla ${ }^{1}$, \\ José Cayotopa ${ }^{2}$, Cristian Hoban ${ }^{2}$, Pedro Ortiz ${ }^{2}$, Arif Mustafa ${ }^{3}$
}

\section{Resumen}

Se realizó un estudio para determinar los efectos del balance electrolítico dietario (BED) sobre el rendimiento productivo, características de la canal y metabolitos sanguíneos del cuy en una granja experimental de Cajamarca, Perú. Se utilizaron 150 cuyes machos distribuidos en cinco tratamientos con cinco niveles de BED: 100, 200, 300, 400 y $500 \mathrm{mEq} / \mathrm{kg}$. Cada tratamiento tuvo seis repeticiones y cada repetición con cinco cuyes/ poza. Los cuyes consumieron alimento concentrado durante 42 días. Al término del periodo experimental se sacrificaron seis cuyes por tratamiento para determinar el rendimiento de carcasa, pesos relativos de las vísceras y concentración de metabolitos sanguíneos. Se encontraron diferencias $(\mathrm{p}<0.05)$ en el peso corporal final, ganancia de peso e índice de conversión alimenticia a favor de cuyes con BED de $300 \mathrm{mEq} / \mathrm{kg}$. No hubo diferencias significativas entre tratamientos en las características de la canal ni en los valores de metabolitos en sangre, excepto la creatinina. Según los resultados de este estudio, el cuy podría ser alimentado con $300 \mathrm{mEq} / \mathrm{kg}$ de BED, con resultados satisfactorios de crecimiento.

Palabras clave: sodio, potasio, cloro, productividad animal, carne

\footnotetext{
${ }^{1}$ Facultad de Ingeniería en Ciencias Pecuarias, Universidad Nacional de Cajamarca, Perú

${ }^{2}$ Facultad de Ciencias Veterinarias, Universidad Nacional de Cajamarca, Perú

${ }^{3}$ McGill University, Mcdonald Campus, QC, Canadá

${ }^{4}$ E-mail:mepaunc@gmail.com; mparedes@unc.edu.pe
}

Recibido: 5 de julio de 2020

Aceptado para publicación: 24 de diciembre de 2020

Publicado: 24 de abril de 2021 
A study was carried out to determine the effects of dietary electrolyte balance (BED) on the productive performance, carcass characteristics and blood metabolites of guinea pig in an experimental farm in Cajamarca, Peru. In total, 150 male guinea pigs distributed in five treatments with five levels of BED: 100, 200, 300, 400 and $500 \mathrm{mEq} / \mathrm{kg}$. Each treatment had six repetitions and each repetition with five guinea pigs/pen. The guinea pigs consumed concentrated feed for 42 days. At the end of the experimental period, six guinea pigs per treatment were slaughtered to determine the carcass yield, relative weights of the viscera and concentration of blood metabolites. Differences $(\mathrm{p}<0.05)$ were found in final body weight, body weight gain, and feed conversion index in favour of guinea pigs with BED of $300 \mathrm{mEq} / \mathrm{kg}$. There were no significant differences between treatments in carcass characteristics or in blood metabolite values, except creatinine. According to the results of this study, guinea pig could be fed $300 \mathrm{mEq} / \mathrm{kg}$ of $\mathrm{BED}$, with satisfactory growth results.

Key words: sodium, potassium, chloride, animal productivity, meat

\section{INTRODUCCIÓN}

Los piensos balanceados para producción animal generalmente cubren el requerimiento de minerales, como $\mathrm{Ca}, \mathrm{P}, \mathrm{Na}$ y $\mathrm{Cl}$; sin embargo, no consideran el balance electrolítico de la dieta (BED), que es el saldo neto entre cationes fijos y aniones (Guzmán-Pino et al., 2015). El BED se determina de acuerdo con el porcentaje de $\mathrm{Na}$, $\mathrm{K}$ y Cl del alimento y el peso molecular de estos elementos, que permiten expresar el BED en $\mathrm{mEq}$ por kilogramo de dieta (Adedokun et al., 2017). El BED regula la homeostasis y mantiene el contenido de electrolitos corporales en equilibrio (Borges et al., 2003a); así, las pérdidas de aminoácidos endógenos en el íleon están significativamente afectadas por BED (Adedokun y Applegate, 2014). La reducción del BED puede influenciar negativamente en la digestibilidad de algunos aminoácidos (Chrystal et al., 2020).

La ingesta de $\mathrm{Na}$ y $\mathrm{K}$ influyen en la cantidad de urea excretada en la orina y el volumen de orina (Burgos et al., 2010). El Cl también forma parte de las secreciones gástricas e influye sobre la actividad del sistema ductal del páncreas que secreta grandes volúmenes de líquido alcalino que contiene concentraciones de $\mathrm{HCO}_{3}$ (Yamaguchi et al., 2017). $\mathrm{El} \mathrm{Na}, \mathrm{K}$ y Cl en la dieta permiten un buen funcionamiento orgánico y crecimiento por ser esenciales para la síntesis de proteínas tisulares y en las reacciones enzimáticas (Borges et al., 2003b). El cloruro de sodio $(\mathrm{NaCl})$ es un componente importante del compartimento extracelular y es el principal determinante de la osmolalidad plasmática y del volumen de líquido extracelular, e influye en la ingestión y excreción urinaria de agua y electrolitos; así en mamíferos, un aumento mínimo en la osmolalidad plasmática de 1 a $2 \%$, disminución en el volumen de líquido extracelular y reducción del $10 \%$ en el volumen sanguíneo inducen sed intensa (Antunes-Rodrígues et al., 2004).

El organismo animal tiene dos compartimentos líquidos principales donde se produce el equilibrio ácido-base: el intracelular y el extracelular, encontrándose en este últi- 
mo el líquido intersticial, plasmático y transcelular (Church et al., 2003). La regulación precisa del volumen y la osmolalidad de los fluidos corporales es fundamental para la supervivencia, y se da principalmente mediante el sistema respiratorio y sistema renal (Hopkins y Sharma 2019). El equilibrio hídrico corporal a nivel renal sostiene la osmolalidad en plasma y la concentración de $\mathrm{Na}$ en orina (Hew-Butler et al., 2014). Alteraciones en el equilibrio de líquidos y electrolitos pueden deberse a una alta pérdida de agua, problemas renales, expansión persistente del compartimento de líquido extracelular y retención de líquido intersticial, provocando problemas nerviosos, cardiacos y respiratorios (Elstgeest et al., 2010).

En cerdos, aves y vacas lecheras se han realizado diversas investigaciones acerca del BED. En lechones, se evaluaron dietas con 16 y $269 \mathrm{mEq} / \mathrm{kg}$ de BED con la finalidad de definir la preferencia de alimentos y cambios en el rendimiento productivo (Guzmán-Pino et al., 2015), en tanto que Deng et al. (2020) encontró que dietas con $250 \mathrm{mEq} / \mathrm{kg}$ de BED redujo el $\mathrm{pH}$ gastrointestinal y promovió el enriquecimiento de la microbiota intestinal con bacterias benéficas. De otra parte, no se disponen de requerimientos de $\mathrm{Cl}$ y $\mathrm{Na}$ en la dieta de cuyes, y los requerimientos de $\mathrm{K}$ del cuy dependen de las concentraciones dietéticas de Ca, P y Mg (NRC, 1995); sin embargo, cuando el cuy es alimentado solo con alfalfa verde, es decir $0.10 \%$ de $\mathrm{Na}, 0.65 \%$ de $\mathrm{Cl}$ y $2.35 \%$ de $\mathrm{K}$ (NRC, 2001), el cuy consume un alimento con un BED estimado de 500 $\mathrm{mEq} / \mathrm{kg}$ y un exceso diario de agua de $53 \mathrm{ml}$ (Chauca, 2018).

La temperatura ambiente y la dieta pueden alterar el equilibrio ácido base del organismo (Borges et al., 2004). El cuy en la sierra peruana es criado a bajas temperaturas ambientales y en condiciones de hipoxia natural que podrían afectar el crecimiento rápido por anaerobiosis celular e hipoxia del tejido muscular (Huchzermeyer, 2012), de alta demanda de oxígeno y que en condiciones adversas, puede conllevar a posibles desor- denes metabólicos (Shlosberg, 1998). En estas condiciones, es importante la regulación de las condiciones de crianza y la oferta de alimentos con un adecuado BED, el cual puede producir cambios en el contenido de oxígeno y pH de la sangre (Dersjant-Li et al., 2002). Del mismo modo se ha demostrado en algunos animales que el BED puede modificar la calidad de la carne, por la caída del $\mathrm{pH}$ post mortem, que junto al nerviosismo y estrés del cuy podría agotar la concentración de glucógeno provocando disminución rápida del pH muscular (Sánchez-Macías et al., 2018). En el presente estudio se propuso encontrar el mejor BED a fin de mejorar la eficiencia del cuy con fines cárnicos criados en condiciones hipóxicas naturales, para lo cual se evaluó el efecto de cinco diferentes niveles de BED sobre indicadores de crecimiento, características de la carcasa y metabolitos sanguíneos.

\section{Materiales y Métodos}

\section{Localización del Estudio}

El presente estudio se realizó entre mayo y junio de 2019 en la granja experimental de animales menores de la Facultad de Ingeniería en Ciencias Pecuarias de la Universidad Nacional de Cajamarca, ubicada a una altitud de $2684 \mathrm{msnm}$, en la provincia de Cajamarca, sierra norte del Perú. La zona presentó temperaturas ambientes mínimas y máximas para la indicada temporada de 5.1 y $20.3{ }^{\circ} \mathrm{C}$, respectivamente, y 12 horas de luz natural.

\section{Animales, Diseño Experimental y Dietas}

Se utilizaron 150 cuyes machos de la raza Perú, de 4-10 semanas de edad, que fueron distribuidos en cinco tratamientos bloqueados por peso. Los animales se alojaron en 30 pozas (seis por tratamiento) con cinco cuyes por poza, las cuales estaban equipadas con un bebedero y un comedero. Los cuyes fueron alimentados ad libitum durante 42 días 
experimentales con cinco piensos isonitrogenados e isoenergéticos, diferenciados por el BED: 100, 200300,400 y $500 \mathrm{mEq} / \mathrm{kg}$. El BED fue calculado considerando las concentraciones de $\mathrm{Na}, \mathrm{K}$ y $\mathrm{Cl}$ de la dieta y sus respectivos pesos moleculares, de acuerdo con la fórmula indicada por Chrystal et al. (2020): $\mathrm{BED}(\mathrm{mEq} / \mathrm{kg})=(\mathrm{mg} / \mathrm{kg}$ de Na$/ 23)$ $+(\mathrm{mg} / \mathrm{kg}$ de $\mathrm{K} / 39.1)-(\mathrm{mg} / \mathrm{kg}$ de $\mathrm{Cl} / 35.5)$.

El BED se ajustó mediante inclusiones dietarias de cloruro de sodio, bicarbonato de sodio y cloruro de calcio como se muestra en el Cuadro 1. Se suministró agua ad libitum, y con la ayuda de dos vasos de precipitado de vidrio borosilicatado de $250 \mathrm{ml}$, se midió el suministro de agua y el residuo en cada bebedero de arcilla cocida tipo pocillo, lo que permitió determinar la ingesta diaria de agua mediante la diferencia entre suministro menos residuo multiplicado por el factor 0.912 que representa la eficiencia en consumo para este tipo de bebedero (Sánchez et al., 2013). Se registró la ingesta diaria de agua permitiendo calcular la ingesta total/cuy según tratamiento y el promedio diario para la etapa experimental.

\section{Rendimiento Productivo}

- El consumo de alimento se registró diariamente por cada poza como la diferencia entre la cantidad de alimento suministrado y el alimento restante al final de cada día.

- Los animales se pesaron en grupo cada semana y la ganancia media diaria (GMD) se determinó como la diferencia entre el peso vivo (PV) inicial y final dividido por 42 días.

- La ingesta diaria de alimento (IDA) de determinó mediante la suma del consumo total de alimento por poza durante el periodo experimental dividido por 42 días y entre el número de cuyes por poza. La IDA se expresa en gramos de materia seca (MS) promedio por cuy, para lo cual se multiplicó el porcentaje de MS de cada dieta experimental por la cantidad de alimento ingerido.
- $\quad$ El índice de conversión alimenticia (ICA) se calculó a partir del consumo promedio de alimento colectivo y el aumento de peso corporal grupal, según poza.

Las mediciones del PV y del suministro y rechazo del alimento se hizo con una balanza electrónica de precisión KERN, de capacidad $2100 \mathrm{~g}$, precisión de lectura $0.01 \mathrm{~g}$.

\section{Carcasa y Vísceras}

Al final del estudio, seis cuyes por tratamiento fueron elegidos al azar y sacrificados mediante corte de la arteria carótida y vena yugular de acuerdo con SánchezMacías et al. (2018). El peso y el pH de la carcasa caliente (CC) se determinaron a los 45 minutos post mortem y de la carcasa fría (CF) luego de $24 \mathrm{~h}$ de refrigeración a $4{ }^{\circ} \mathrm{C}$. La medición del $\mathrm{pH}$ se realizó con un pHmetro digital portátil Hanna ${ }^{\circledR}$ HI 99163 en el músculo Psoas mayor. Además, se extrajeron las vísceras de la $\mathrm{CC}$ y se pesaron utilizando la balanza indicada anteriormente. El rendimiento de carcasa $(\mathrm{RC})$ se determinó mediante el peso de la CC x 100 dividido por el PV final.

El peso de cada carcasa caliente $\left(\mathrm{P}_{0}\right)$ se utilizó para determinar la pérdida por goteo de la carne mediante la diferencia de peso con la carcasa $\left(\mathrm{P}_{\mathrm{f}}\right)$ refrigerada: $\% \mathrm{PG}=100$ $\left(\left(\mathrm{P}_{0} / \mathrm{P}_{\mathrm{f}}\right) \times 100\right)$ (Pang et al., 2020). El tracto gastrointestinal (TGI), hígado, corazón, pulmones, grasa peri-renal, riñones y cabeza fueron removidos de la carcasa, y pesados en una balanza Kern de $400 \mathrm{~g}$ de capacidad $( \pm 0.01 \mathrm{~g})$. Los pesos de las vísceras se relacionaron al peso vivo de cada cuy según tratamientos mediante la división del peso del órgano x 100 sobre el PV.

\section{Metabolitos del Suero Sanguíneo}

El análisis de sangre fue realizado en el Laboratorio de Nutrición Crampton del Departamento de Ciencia Animal, Universidad McGill, Canadá. Se seleccionó un cuy con el peso más cercano al promedio de cada poza 
Cuadro 1. Ingredientes y contenido nutricional de las dietas experimentales $(\mathrm{g} / \mathrm{kg}$, base fresca) con diferente balance electrolítico ( $\mathrm{mEq} / \mathrm{kg})$

\begin{tabular}{|c|c|c|c|c|c|}
\hline & \multicolumn{5}{|c|}{ Balance Electrolítico de la Dieta (BED), (mEq/kg) } \\
\hline & 100 & 200 & 300 & 400 & 500 \\
\hline \multicolumn{6}{|l|}{ Ingredientes } \\
\hline Maíz & 200 & 200 & 200 & 200 & 200 \\
\hline Afrecho de trigo & 326 & 328 & 328 & 324 & 316 \\
\hline Torta de soya & 190 & 190 & 190 & 190 & 190 \\
\hline Polvillo de arroz & 200 & 200 & 200 & 200 & 200 \\
\hline Heno de alfalfa & 60 & 60 & 60 & 60 & 60 \\
\hline Carbonato de calcio & 3 & 8 & 14 & 15 & 15 \\
\hline Sal común & 3 & 3 & 3 & - & - \\
\hline DL Metionina & 1 & 1 & 1 & 1 & 1 \\
\hline $\begin{array}{l}\text { Premezcla vitamínica- } \\
\text { mineral }^{1}\end{array}$ & 1 & 1 & 1 & 1 & 1 \\
\hline Ascorbil oral $^{2}$ & 1 & 1 & 1 & 1 & 1 \\
\hline Bicarbonato de sodio & 2 & 1 & 1 & 8 & 16 \\
\hline Cloruro de calcio & 13 & 7 & 1 & - & - \\
\hline Total & 1000.0 & 1000.0 & 1000.0 & 1000.0 & 1000.0 \\
\hline \multicolumn{6}{|c|}{ Contenido nutricional calculado } \\
\hline Materia seca & 881.5 & 881.4 & 881.3 & 881.9 & 882.8 \\
\hline Proteína cruda & 182.9 & 183.1 & 183.1 & 182.2 & 181.0 \\
\hline $\begin{array}{l}\text { Energía digestible, } \\
\mathrm{Kcal} / \mathrm{kg}\end{array}$ & 2871 & 2874 & 2874 & 2859 & 2839 \\
\hline Fibra & 94.1 & 94.2 & 94.2 & 93.5 & 92.5 \\
\hline Lisina & 9.2 & 9.2 & 9.2 & 9.1 & 9.1 \\
\hline Metionina & 3.6 & 3.6 & 3.6 & 3.6 & 3.6 \\
\hline Calcio & 7.8 & 7.6 & 7.8 & 7.8 & 7.8 \\
\hline Fósforo & 7.7 & 7.7 & 7.7 & 7.7 & 7.6 \\
\hline Sodio & 2.0 & 1.7 & 1.7 & 2.4 & 4.6 \\
\hline Cloro & 10.9 & 7.1 & 3.3 & 0.9 & 0.9 \\
\hline Potasio & 13 & 13 & 13 & 13 & 13 \\
\hline $\begin{array}{l}\text { Balance electrolítico, } \\
\mathrm{mEq} / \mathrm{kg}^{3}\end{array}$ & 104 & 200 & 305 & 404 & 496 \\
\hline \multicolumn{6}{|c|}{$\begin{array}{l}\text { Cada kilogramo contiene: Vit. A } 9000 \text { mil UI, Vit. D3 } 2500 \text { mil UI, Vit. E } 15000 \text { UI, Vit. Kз } 2.5 \\
\text { g, tiamina } 1.5 \mathrm{~g} \text {, riboflavina } 6.5 \mathrm{~g} \text {, cianocobalamina } 0.01 \mathrm{~g} \text {, ácido pantoténico } 5.50 \mathrm{~g} \text {, ácido } \\
\text { fólico } 1 \mathrm{~g} \text {, niacina } 25 \mathrm{~g}, \mathrm{Mn} 70 \mathrm{~g}, \mathrm{Zn} 70 \mathrm{~g}, \mathrm{Fe} 30 \mathrm{~g}, \mathrm{Cu} 8 \mathrm{~g}, \mathrm{I} 1 \mathrm{~g} \text {, Se } 0.30 \mathrm{~g}, \mathrm{Co} 0.1 \mathrm{~g} \\
\text { Fuente de ácido ascórbico } 100 \%\end{array}$} \\
\hline \multicolumn{6}{|l|}{$(\mathrm{meq} / \mathrm{kg})=(\mathrm{mg} / \mathrm{kg} \mathrm{iva}$} \\
\hline
\end{tabular}


Cuadro 2. Efecto del balance electrolítico dietario sobre indicadores de crecimiento de cuyes (Cavia porcellus) evaluado entre 29 y 70 días de edad

\begin{tabular}{lccccccc}
\hline & \multicolumn{5}{c}{ Balance electrolítico $(\mathrm{mEq} / \mathrm{kg})$} & \multirow{2}{*}{ SEM $^{1}$} & \multirow{2}{*}{$p$} \\
\cline { 2 - 5 } & 100 & 200 & 300 & 400 & 500 & & \\
\hline PC inicial (g) & 429.7 & 427.4 & 441.1 & 436.6 & 440.9 & 2.83 & 0.796 \\
PC final (g) & $764.9^{\mathrm{b}}$ & $779.4^{\mathrm{b}}$ & $861.2^{\mathrm{a}}$ & $801.4^{\mathrm{b}}$ & $691.5^{\mathrm{c}}$ & 27.49 & 0.042 \\
IDA (g de & 50.1 & 49.4 & 51.7 & $51.4^{2}$ & 49.3 & 0.48 & 0.671 \\
MS/cuy) & & & & & & & \\
GMD (g/cuy) & $8.0^{\mathrm{b}}$ & $8.4^{\mathrm{b}}$ & $10.0^{\mathrm{a}}$ & $8.7^{\mathrm{b}}$ & $6.0^{\mathrm{c}}$ & 0.65 & 0.039 \\
ICA & $6.2^{\mathrm{b}}$ & $5.9^{\mathrm{b}}$ & $5.2^{\mathrm{c}}$ & $5.9^{\mathrm{b}}$ & $8.2^{\mathrm{a}}$ & 0.51 & 0.027 \\
CDA (ml/cuy) & $101.4^{\mathrm{c}}$ & $102.9^{\mathrm{c}}$ & $110.3^{\mathrm{b}}$ & $118.9^{\mathrm{ab}}$ & $138.4^{\mathrm{a}}$ & 6.76 & 0.041 \\
\hline
\end{tabular}

$a, b, c$ Valores medios en la misma fila con diferente superíndice son estadísticamente diferentes $(\mathrm{p}<0.05)$

${ }^{1}$ Error estándar de la media

PC: peso corporal; IDA: ingesta diaria de alimento; GMD: ganancia media diaria; ICA: índice de conversión alimenticia; CDA: consumo diario de agua

y se desangraron a través del corte de la arteria carótida y de la vena yugular. La sangre se recogió en tubos con heparina y se centrifugó inmediatamente a $2000 \mathrm{rpm}$ durante 15 minutos a $10^{\circ} \mathrm{C}$. El plasma resultante se colocó en dos tubos Eppendorf de $1.5 \mathrm{ml}$ y se almacenaron a $-20^{\circ} \mathrm{C}$.

La actividad sérica de aspartato aminotransferasa (AST) y fosfatasa alcalina (ASP), la proteína total (PT), el nitrógeno ureico en sangre (NUS) y el contenido de albúmina fueron determinados con el analizador bioquímico Yellow Springs Instrument YSI 2300 (Life Sciences). La concentración de $\mathrm{Na}$ y K en plasma se midió utilizando electrodos selectivos de iones, el cloro se estimó utilizando un espectrofotómetro de absorción atómica Biobase BK-AA32ON. La osmolalidad plasmática (PO) se determinó utilizando un osmómetro de presión de vapor VAPRO 5520.

\begin{abstract}
Análisis Estadístico
Los datos se analizaron mediante el procedimiento MIXED del SAS (2006). Los datos de rendimiento del crecimiento, carcasas y los metabolitos del suero sanguíneo obtenidos se analizaron bajo un diseño de bloques completos al azar. Se usó la prueba de Duncan para detectar diferencias entre las medias de los tratamientos, y se declaró la significación estadística a un nivel de $\mathrm{p}<0.05$.
\end{abstract}

\section{Resultados y Discusión}

\section{Rendimiento Productivo}

Los valores medios para el peso corporal (PC), la ganancia media diaria, la ingesta de alimento e índice de conversión alimenticia de los cuyes se resumen en el Cuadro 2. Hubo diferencias significativas $(\mathrm{p}<0.05)$ para PC final, GMD e ICA a favor del BED con 
$300 \mathrm{mEq} / \mathrm{kg}$. El peso corporal se encuentra dentro del rango reportado por Kouakou et al. (2013), y fue superior al peso de otros genotipos de cuyes reportados por Chauca (1995).

Los niveles de BED menores y mayores de $300 \mathrm{mEq} / \mathrm{kg}$ afectaron los parámetros productivos de los cuyes; sin embargo, no generaron un mayor consumo de alimento concentrado, aunque influenciaron a un mayor consumo de agua en los grupos BED de 400 y $500 \mathrm{mEq} / \mathrm{kg}$. El rendimiento obtenido por efecto del BED, en condiciones de frío y altitud, sugiere que el cuy es una especie muy susceptible a cambios electrolíticos de la dieta; lo cual no siempre sucede con pollos alimentados con BED variados (Adedokun et al., 2017). Asimismo, se concuerda con Ravindran et al. (2008) quienes indican que BED inferiores a $225 \mathrm{mEq} / \mathrm{kg}$ y mayores a $300 \mathrm{mEq} / \mathrm{kg}$ produjeron mermas en el crecimiento de pollos parrilleros debido a la reducción de la digestibilidad de los aminoácidos dietarios.

Las dietas experimentales se diseñaron para generar diferentes valores de BED, variando las fuentes de calcio y de sodio en la dieta basal de cada tratamiento. Para reducir el valor de BED, el carbonato de calcio fue reemplazado por cloruro de calcio, manteniendo una concentración constante de calcio en las dietas (entre 7.6 a $7.8 \mathrm{~g} / \mathrm{kg}$ ), pero con altas concentraciones de cloruro en los tratamientos con 100 y $200 \mathrm{mEq} / \mathrm{kg}$ de BED; de esta manera, es posible que las diferencias en la solubilidad entre las sales de cloruro y carbonato puedan haber promovido diferencias en la absorción real de calcio (GuzmánPino et al., 2015), generando diferencias en el crecimiento de los cuyes.

El menor crecimiento de los cuyes también podría explicarse por los altos niveles de $\mathrm{Cl}$ en las dietas con menor BED, dado el efecto antagonista del $\mathrm{Cl}$ sobre la calcificación del sistema esquelético (Lázaro et al., 2002). Por otro lado, se suministró bicarbonato de sodio para aumentar el BED de los tratamientos con 400 y $500 \mathrm{mEq} / \mathrm{kg}$, reemplazando al cloruro de sodio, lo cual pudo afectar el crecimiento en estos animales, dado que el sodio es el principal catión del líquido extracelular y el regulador más importante de la osmolalidad sanguínea. Al aumentar la osmolalidad, las neuronas hipotalámicas sufren una disminución en su volumen por causas osmóticas y, a consecuencia de este efecto, se estimula la sed y se libera vasopresina con el fin de incrementar la ingesta de agua y retención de la misma en el túbulo colector renal a fin de disminuir la osmolalidad (Paniagua, 2015). Esto explica, que el mejor BED para cuyes en crecimiento estuvo en $300 \mathrm{mEq} / \mathrm{kg}$, porque dietas con menores BED fueron acidogénicas, provocando posiblemente disminución de $\mathrm{pH}$ sanguíneo. Por el contrario, dietas con altos BED provocaron posiblemente hipernatremia con los subsiguientes trastornos como mayor consumo de agua, incremento de la osmolalidad y mayor esfuerzo renal; todo lo cual se refleja en menores GMD e ICA encontrados en el presente estudio. Tanto la acidosis como la alcalosis son estados patológicos según su severidad; para lo cual el organismo mediante mecanismos homeostáticos regula el balance ácido-base afectado por la ingesta de sustancias contenidas en el alimento (Caballero y Clerici, 2020).

\section{Características de Carcasa}

Los valores medios para las características de canal de los animales por tratamiento se presentan en el Cuadro 3. Se encontraron diferencias estadísticas $(\mathrm{p}<0.05)$ entre pesos corporales y pesos de carcasa. Los pesos de la carcasa y rendimiento de carcasa estuvieron dentro de los rangos reportados (Mínguez y Calvo, 2018; Sánchez-Macías et al., 2018; Mínguez et al., 2019).

La diferencia de pesos de la carcasa entre tratamientos se debió al peso vivo final de los cuyes, sin encontrarse diferencias estadísticas en el peso relativo de la carcasa 
Cuadro 3. Efecto del balance electrolítico dietario sobre las características de la carcasa de cuyes (Cavia porcellus) de 70 días de edad

\begin{tabular}{|c|c|c|c|c|c|c|c|}
\hline & \multicolumn{5}{|c|}{ Balance electrolítico (mEq/kg) } & \multirow{2}{*}{$\mathrm{SEM}^{1}$} & \multirow{2}{*}{$p$} \\
\hline & 100 & 200 & 300 & 400 & 500 & & \\
\hline PC de sacrificio $(\mathrm{g})$ & 766 & 899 & 902 & 802 & 712 & 37.28 & 0.046 \\
\hline $\operatorname{PCC}(g)$ & 538 & 616 & 661 & 545 & 483 & 31.31 & 0.049 \\
\hline PCF (g) & 520 & 594 & 638 & 524 & 465 & 29.35 & 0.045 \\
\hline $\mathrm{RC}(\%)$ & 70,3 & 68.7 & 73.1 & 67.9 & 68.1 & 1.09 & 0.542 \\
\hline $\mathrm{pH} 45 \mathrm{~m}$ & 6.9 & 6.9 & 7.1 & 7.1 & 7.2 & 0.06 & 0.831 \\
\hline $\mathrm{pH} 24 \mathrm{~h}$ & 6.4 & 6.5 & 6.5 & 6.7 & 6.7 & 0.06 & 0.884 \\
\hline PPG $(\%)$ & 3.3 & 3,5 & 3.4 & 3.8 & 3.7 & 0.11 & 0.524 \\
\hline \multicolumn{8}{|c|}{ Peso de menudencias (g/100 g de PC) } \\
\hline TGI & 23.3 & 22.2 & 20.6 & 21.1 & 21.5 & 0.47 & 0.411 \\
\hline Hígado & 2.9 & 3.0 & 2.8 & 2.9 & 3.1 & 0.04 & 0.807 \\
\hline Corazón & 0.4 & 0.3 & 0.4 & 0.5 & 0.5 & 0.03 & 0.863 \\
\hline Cabeza & 8.8 & 7.5 & 7.7 & 7.6 & 7.7 & 0.24 & 0.445 \\
\hline Riñones & 1.2 & 1.0 & 0.8 & 1 & 1.2 & 0.07 & 0.118 \\
\hline Pulmones & 0.9 & 0.7 & 0.6 & 0.7 & 0.8 & 0.04 & 0.814 \\
\hline Grasa perineal & 0.2 & 0.3 & 0.2 & 0.2 & 0.2 & 0.01 & 0.911 \\
\hline \multicolumn{8}{|c|}{$\begin{array}{l}\text { ( } p<0.05) \\
{ }^{a} \text { SEM: Error estándar de la media }\end{array}$} \\
\hline
\end{tabular}

respecto del peso corporal. El $\mathrm{pH}$ y las pérdidas por goteo de las canales de cuy no mostraron diferencias atribuidas a los diferentes niveles de BED. El pH de las carcasas fue similar a los valores 6.74 y 5.96 medidos en el músculo Longissimus dorsi, a 1 y $24 \mathrm{~h}$ post mortem reportados por Lucas et al. (2017) en cuyes alimentados con $80 \%$ de forraje verde más afrecho de trigo en el valle altoandino de El Mantaro; asimismo, Nakandakari et al. (2014) encontraron valores de $\mathrm{pH}$ en el músculo Psoas mayor de cuy de 6.71 y 6.06 a las 0 y $24 \mathrm{~h}$ post mortem, respectivamente, a partir de cuyes alimenta- dos con maíz chala y alimento balanceado, en condiciones de costa central del Perú. Por tanto, las pequeñas diferencias de $\mathrm{pH}$ del cuy pueden atribuirse a condiciones ambientales de crianza y posiblemente al músculo donde se midió el pH.

El peso relativo de las vísceras no fue influenciado por los niveles de BED. Los pesos relativos de las vísceras concuerdan con los hallados por Mustafa et al. (2019) en cuyes del valle de Condebamba, región de Cajamarca. 
Cuadro 4. Efecto del balance electrolítico dietario sobre los metabolitos séricos de la sangre cuyes (Cavia porcellus) de 70 días de edad

\begin{tabular}{|c|c|c|c|c|c|c|c|}
\hline \multirow{2}{*}{ Metabolitos séricos } & \multicolumn{5}{|c|}{ Balance electrolítico (mEq/kg) } & \multirow{2}{*}{ SEM $^{1}$} & \multirow{2}{*}{$p$} \\
\hline & 100 & 200 & 300 & 400 & 500 & & \\
\hline$\overline{\mathrm{AST}}(\mathrm{U} / \mathrm{l})$ & 70.7 & 73.3 & 85.3 & 83.4 & 86.3 & 3.24 & 0.076 \\
\hline Albúmina $(\mathrm{g} / \mathrm{Ll}$ & 28.3 & 26.7 & 25.7 & 27.7 & 29.4 & 0.64 & 0.099 \\
\hline ALP (U/Ll & 163.3 & 199 & 175 & 187.2 & 198.1 & 6.86 & 0.145 \\
\hline Creatinina $(\mu / \mathrm{Ll}$ & $107^{\mathrm{c}}$ & $123^{\mathrm{bc}}$ & $150^{\mathrm{b}}$ & $182^{\mathrm{ab}}$ & $201^{\mathrm{a}}$ & 17.56 & 0.042 \\
\hline $\begin{array}{l}\text { Osmolalidad } \\
\left(\mathrm{mOsm} / \mathrm{kg} \mathrm{H}_{2} \mathrm{O}\right)\end{array}$ & 301.3 & 297.7 & 318.3 & 322.1 & 325.7 & 5.67 & 0.331 \\
\hline Proteína total (g/l) & 48.7 & 46.3 & 47.7 & 48.9 & 49.1 & 0.52 & 0.884 \\
\hline $\begin{array}{l}\text { Nitrógeno ureico } \\
(\mathrm{mmol} / \mathrm{l})\end{array}$ & 10.3 & 9.7 & 9.1 & 9.8 & 10.7 & 0.27 & 0.524 \\
\hline $\mathrm{Na}(\mathrm{mmol} / \mathrm{l})$ & 141.3 & 142 & 143.3 & 145.3 & 145.9 & 0.89 & 0.759 \\
\hline $\mathrm{K}(\mathrm{mmol} / \mathrm{l})$ & 7.7 & 7 & 6.2 & 6.8 & 6.2 & 0.28 & 0.127 \\
\hline $\mathrm{Cl}(\mathrm{mmol} / \mathrm{l})$ & 103.7 & 103.3 & 101 & 102.3 & 103.1 & 0.48 & 0.672 \\
\hline $\mathrm{Na}: \mathrm{K}$ & 18.3 & 20.3 & 23.3 & 21.4 & 23.5 & 0.97 & 0.185 \\
\hline
\end{tabular}

\section{Metabolitos Sanguíneos}

Los parámetros sanguíneos se presentan en el Cuadro 4. Los niveles de AST, albúmina, ALP, osmolalidad, PT, NUS y minerales no difirieron significativamente entre los tratamientos por efecto de BED, excepto la creatinina $(p<0.05)$. AST es una enzima importante para evaluar la función hepática, ya que sus niveles plasmáticos reflejan el estado de salud del hígado. Aumentos de este metabolito generalmente se asocian con fugas del citoplasma de las células lesionadas o como resultado de una mayor síntesis de gamma glutamiltransferasa (Zeng et al., 2014). Los niveles séricos similares de AST y ALP con el aumento de la inclusión de BED son indicadores de que los niveles altos de BED no afectaron negativamente las funciones hepáticas.

Los índices bioquímicos en suero normalmente reflejan la condición de un animal, así como cambios en respuesta a factores internos y exógenos (Toghyani et al., 2010). La enfermedad renal ocurre con mayor frecuencia en cuyes mayores de 12 meses, presentando signos clínicos como pérdida de peso, polidipsia, oliguria o poliuria y muerte inesperada; encontrándose valores elevados de creatinina y tamaño disminuido del riñón a la necropsia (Holowaychuk, 2006). En el presente estudio, los cuyes solo llegaron a una edad de 70 días y los niveles de creatinina 
estuvieron ligeramente por encima del rango normal (53- $194 \mu \mathrm{mol} / \mathrm{l})$ en aquellos que consumieron alimento con BED de $500 \mathrm{mEq} / \mathrm{kg}$.

\section{Conclusiones}

- El balance electrolítico dietario entre 100 y $500 \mathrm{mEq} / \mathrm{kg}$ en cuyes no generó signos clínicos de enfermedad o muerte, pero afectó los indicadores de crecimiento.

- El nivel de balance electrolítico de la dieta con $300 \mathrm{mEq} / \mathrm{kg}$ podría mejorar positivamente los indicadores de crecimiento del cuy sin afectar negativamente los indicadores relativos de la carcasa y la función hepática y renal.

\section{Literatura Citada}

1. Adedokun SA, Pescatore AJ, Ford MJ, Jacob JP, Helmbrecht A. 2017. Examining the effect of dietary electrolyte balance, energy source, and length of feeding of nitrogen-free diets on ileal endogenous amino acid losses in broilers. Poultry Sci 96: 3351-3360. doi: 10.3382/ps/pex159

2. Adedokun SA, Applegate TJ. 2014. Dietary electrolyte balance influences ileal endogenous amino acid losses in broiler chickens. Poultry Sci 93: 935-942. doi: 10.3382/ps.2013-03661

3. Antunes-Rodrigues J, De Castro M, Elias LLK, Valenca V, McCann SM. 2004. Neuroendocrine control of body fluid metabolism. Physiol Rev 84: 169208. doi: 10.1152/physrev.00017.2003

4. Borges SA, Fischer da Silva AV, Majorka A, Hooge DM, Cummings KR. 2004. Physiological responses of broiler chickens to heat stress and dietary electrolyte balance (sodium plus potassium minus chloride, milliequivalents per kilogram). Poultry Sci 83: 1551-1558. doi: $10.1093 / \mathrm{ps} / 83.9 .1551$
5. Borges $S A$, Fischer da Silva AV, Ariki J, Hooge DM, Cummings KR. $2003 a$. Dietary electrolyte balance for broiler chickens under moderately high ambient temperatures and relative humidities. Poultry Sci 82: 301-308. doi: 10.1093/ps/ 82.2.301

6. Borges $S A$, Fischer da Silva AV, Ariki J, Hooge DM, Cummings KR. 2003 b. Dietary electrolyte balance for broiler chickens exposed to thermoneutral or heat-stress environments. Poultry Sci 82: 428-435. doi: $10.1093 / \mathrm{ps} / 82.3 .428$

7. Burgos SA, Embertson NM, Zhao Y, Mitloehner FM, De Peters EJ, Fadel JG. 2010. Prediction of ammonia emission from dairy cattle manure based on milk urea nitrogen: relation of milk urea nitrogen to ammonia emissions. J Dairy Sci 93: 2377-2386. doi: 10.3168/ jds.2009-2415

8. Caballero AJD, Clerici C. 2020. Dieta alcalina y su relación con la salud y la enfermedad: una revisión sistemática. Actualización en Nutrición 21: 16-24.

9. Chauca L. 1995. Guinea pig (Cavia porcellus) production in the Andean countries. World Anim Rev 83: 9-19.

10. Chauca L. 2018. Manual de crianza de cuyes. Lima, Perú: Instituto Nacional de Innovación Agraria - INIA. 80 p.

11. Chrystal P, Moss AF, Khoddami A, Naranjo VD, Selle PH, Liu SY. 2020. Effects of reduced crude protein levels, dietary electrolyte balance, and energy density on the performance of broiler chickens offered maize-based diets with evaluations of starch, protein, and amino acid metabolism. Poultry Sci 99: 14211431. doi: 10.1016/j.psj.2019.10.060

12. Church D, Pond W, Pond K. 2003. Fundamentos de nutrición y alimentación de animales. $2^{\circ}$ ed. México DF: Limusa. $635 \mathrm{p}$.

13. Deng Q, Shao Y, Wang O, Li J, Li Y, Ding $X$, Huang $P$, et al. 2020. Effects and interaction of dietary electrolyte balance and citric acid on the intestinal function of weaned piglets. J Anim Sci 98: skaa106. doi: 10.1093/jas/skaa106 
14. Dersjant-Li $Y$, Verstegen MWA, Jansman A, Schulze H, Schrama JW, Verreth $J A$. 2002. Changes in oxygen content and acid-base balance in arterial and portal blood in response to the dietary electrolyte balance in pigs during a 9-h period after a meal. J Anim Sci 80: 12331239. doi: $10.2527 / 2002.8051233 x$

15. Elstgeest LE, Martens SE, Lopriore E, Walther FJ, te Pas AB. 2010. Does parenteral nutrition influence electrolyte and fluid balance in preterm infants in the first days after birth? Plos One 5: e9033. doi: 10.1371/journal.pone.0009033

16. Guzmán-Pino SA, Solà-Oriol D, Davin R, Manzanilla EG, Pérez JF. 2015. Influence of dietary electrolyte balance on feed preference and growth performance of postweaned piglets. J Anim Sci 93: 2840-2848. doi: 10.2527/jas2014-8380

17. Hew-Butler T, Hummel J, Rider B, Verbalis JG 2014. Characterization of the effects of the vasopressin V2 receptor on sweating, ûuid balance, and performance during exercise. Am J Physiol Regul Integr Comp Physiol 307: 366-375. doi: 10.1152/ajpregu.00120.2014

18. Holowaychuk M. 2006. Renal failure in a guinea pig (Cavia porcellus) following ingestion of oxalate containing plants. Can Vet J 47: 787-789.

19. Hopkins E, Sharma S. 2019. Physiology, acid base balance. In: Stat Pearls [Internet]. Treasure Island (FL): Available in: https://www.ncbi.nlm.nih.gov/books/NBK507807/

20. Huchzermeyer F. 2012. Broiler ascites: a review of the ascites work done at the poultry section of the Onderstepoort Veterinary Institute 1981-1990. World Poultry Sci J 68: 41-50. doi: 10.1017/ S0043933912000050

21. Kouakou NDV, Grongnet JF, Assidjo $N$, Thys E, Marnet PG, Catheline D, Legrand P, Kouba M. 2013. Effect of a supplementation of Euphorbia heterophylla on nutritional meat quality of Guinea pig (Cavia porcellus L). Meat
Sci 93: 821-826. doi: 10.1016/ j.meatsci.2012.11.036

22. Lázaro R, Mateos GG, La Torre M. 2002. Nutrition and feeding of turkeys for fattening. In: XVIII Specialization Course FEDNA, Spain.

23. Lucas JR, Balcázar-Nakamatsu S, Tirado O, Rodríguez A. 2017. $\mathrm{El} \mathrm{pH} \mathrm{de}$ la carne de cobayo (Cavia porcellus) procedente del manejo deficiente del bienestar animal durante el sacrificio en la sierra central del Perú. FAVE Sección Ciencias Veterinarias 16: 70-73. doi: 10.14409/favecv.v16i2.6818.

24. Mínguez C, Calvo A, Zeas V, SánchezMacías D. 2019. A comparison of the growth performance, carcass traits, and behavior of guinea pigs reared in wire cages and floor pens for meat production. Meat Sci 152: 38-40. doi: 10.1016/ j.meatsci.2019.02.012

25. Mínguez C, Calvo A. 2018. Effect of supplementation with fresh orange pulp (Citrus sinensis) on mortality, growth performance, slaughter traits and sensory characteristics in meat guinea pigs. Meat Sci 145: 51-54. doi: 10.1016/ j. meatsci.2018.06.003

26. Mustafa AF, Chavarri EC, Mantilla JG, Mantilla JO, Paredes, MA. 2019. Effects of feeding flaxseed on performance, carcass trait, and meat fatty acid composition of guinea pigs (Cavia porcellus) under northern Peruvian condition. Trop Anim Health Prod 51: 2611-2617. doi: 10.1007/s11250-01901977-0.

27. Nakandakari L, Gutiérrez, E, Chauca L, Valencia R. 2014. Medición del pH intramuscular del cuy (Cavia porcellus) durante las primeras 24 horas post beneficio tradicional. Salud Tecnol Vet 2: 99-105.

28. [NRC] National Research Council. 1995. Nutrient requirements of laboratory animals. $4^{\text {th }}$ rev. ed. Subcommittee on Laboratory Animal Nutrition. Washington (DC). [Internet]. Available in: https://www.ncbi.nlm.nih.gov/books/ NBK231927/ 
29. [NRC] National Research Council. 2001. Nutrient Requirements of dairy cattle. $7^{\text {th }}$ Rev. ed. Subcommittee on Dairy Cattle Nutrition Committee on Animal Nutrition Board on Agriculture and Natural Resources. Washington DC, USA: NRC. $381 \mathrm{p}$.

30. Pang B, Bowker B, Gamble G, Zhang $J$, Yang $Y$, Yu X, Sun JX, Zhuang $H$. 2020. Muscle water properties in raw intact broiler breast fillets with the woody breast condition. Poultry Sci 99: 46264633. doi: 10.1016/j.psj.2020.05.031

31. Paniagua L. 2015. Hipernatremia: fisiopatología, diagnóstico y tratamiento. Rev Med Costa Rica Centroam 72: 815-817.

32. Ravindran V, Cowieson AJ, Selle PH. 2008. Influence of dietary electrolyte balance and microbial phytase on growth performance, nutrient utilization, and excreta quality of broiler chickens. Poultry Sci 87: 677-688. doi: 10.3382/ps.200700247

33. Sánchez R, Jiménez R, Huamán $H$, Bustamante J, Huamán A. 2013. Respuesta productiva y económica al uso de cuatro tipos de bebederos y a la adición de vitamina $\mathrm{c}$ en la crianza de cuyes en época seca en el valle del Mantaro. Rev Inv Vet Perú 24: 283-292.

34. Sánchez-Macías D, Barba-Maggi L, Morales-de la Nuez A, Palmay-Paredes J. 2018. Guinea pig for meat production: a systematic review of factors affecting the production, carcass and meat quality. Meat Sci 143: 165-176. doi: 10.1016/j.meatsci.2018.05.004

35. Shlosberg, A. 1998. Comparative effect of added chloride, ammonium chloride, or potassium bicarbonate in the drinking water of broilers, and feed restriction, on the development of the ascites syndrome. Poultry Sci 77: 1287-1296. doi: 10.1093/ ps/77.9.1287

36. Toghyani M, Gheisari A, Ghalamkari G, Mohammadrezaei M. 2010. Growth performance, serum biochemistry and blood hematology of broiler chicks fed different levels of black seed (Nigella sativa) and peppermint (Mentha piperita). Livest Sci 129: 173178. doi: 10.1016/j.livsci.2010.01.021

37. Yamaguchi M, Steward MC, Smallbone K, Sohma Y, Yamamoto A, Ko SB, Kondo T, et al. 2017. Bicarbonate-rich fluid secretion predicted by a computational model of guinea-pig pancreatic duct epithelium. J Physiol 15: 1947-1972. doi: 10.1113/JP273306

38. Zeng QF, Yang GL, Liu GN, Wang JP, Bai SP, Ding XM, Luo YH, Zhang $K Y$. 2014. Effects of dietary gossypol concentration on growth performance, blood profiles, and hepatic histopathology in meat ducks. Poultry Sci 93: 20002009. doi: 10.3382/ps.2013-03841 